\title{
A Surrogate Approach to Reveal Microstructural Mechanisms Controlling the 3D Short Crack Growth in a Ti-6Al-4V Alloy
}

\author{
Meysam Hassanipour ${ }^{*} 1$, Shinta Watanabe ${ }^{1},{\operatorname{Han~} \mathrm{Li}^{1} \text {, Kyosuke Hirayama }}^{1}$, Hiroyuki Toda ${ }^{1}$, Akihisa Takeuchi ${ }^{2}$, Kentaro Uesugi $^{2}$ \\ ${ }^{1}$ Department of Mechanical Engineering, Kyushu University, 744, Motooka, Nishi Ward, Fukuoka, Fukuoka 819-0395, Japan \\ 2 Japan Synchrotron Radiation Research Institute, 1-1-1, Kouto, Sayo Cho, Sayo Gun, Hyogo 679-5148, Japan
}

\begin{abstract}
Microstructural features and short crack growth behavior were characterized and linked in a Ti-6Al-4V by employing X-ray micro-tomography combined with EBSD serial sectioning. Statistical analysis was used to rank the contributing features to the crack behavior. Afterwards, by creating surrogate models, the microstructural mechanism controlling the short crack behavior were revealed. Short crack preferably grows inside the predominant $\alpha$ phase above the average microstructural fraction. A high number of grains in contact with cracked $\alpha$ grains elongated in the loading direction may impose a constraint on the crack opening resulting in low crack growth rates. As the crack front becomes larger, the increase in the shear stress field away from the cracked grain leads to crack bifurcations, resulting in a decrease in crack driving forces with low crack growth rates. This leads to a preferable growth in $\alpha+\beta$ phase and along the interface above the average microstructural fractions.
\end{abstract}

\section{Introduction}

There is an ongoing research in order to fabricate alloys with enhanced mechanical properties [1,2]. As the cyclic load is applied, there is a contribution of different microstructural features that leads to a local change in properties. Traditionally, different fabrication process such heat treatment, rolling, etc., are employed in order to make alloys with predominant features (e.g., grain size) to estimate the correlation with local properties (e.g., crack growth) [3-5]. Nonetheless, there is a large variation in local features and properties; thus, large number of specimens are required to estimate the correlation [6,7]. Moreover, extensive local observations are only conducted on the surface of the specimens to identify the controlling micromechanisms $[4,8]$. Therefore, it can be said a large number of specimens are prepared and a large-scale analysis are conducted but there is still a high uncertainty in terms of local behavior and the controlling mechanisms.

In order to reach a 3D, in-situ, and representative analysis, X-ray microtomography can be employed. By using this type of observation in one specimen, a large number of local features and their properties can be linked with $1 \mu \mathrm{m}$ of spatial resolution. It should be said that each feature contributes to the evolution of the local properties with respect to time. In order to rank the contribution of each feature in space and time, there is an essential need for statistical analysis. Furthermore, by creating surrogate models with the most contributing features, a model that can represent the microstructure-property can be build up. As a result, the relationship between features and their properties can be studied and the eventual microstructural mechanisms can be revealed. As a result, a higher certainty is reached and a controlled process that may induce specific microstructural mechanisms can be proposed.

In this study, using X-ray microtomography and EBSD serial sectioning, 3D short crack and microstructural features were reconstructed and linked together. Afterwards, using the global sensitivity and principal component analysis, the contribution of microstructural features controlling the short crack growth were ranked. Then, by creating surrogate models the relationship between the most contributing microstructural features and crack growth behavior were determined and the micromechanisms can be revealed.

\section{Material and experimental procedures}

(C) The Authors, published by EDP Sciences. This is an open access article distributed under the terms of the Creative Commons Attribution License 4.0 (http://creativecommons.org/licenses/by/4.0/). 
The chemical composition and heat treatment of the Ti-6Al-4V alloy employed in this study is given in [9]. At the end of heat treatment, this alloy was composed of $65 \pm 3 \%$ primary $\alpha$ phase and $35 \pm 3 \%$ of $\alpha+\beta$ phase. The primary $\alpha$ mean grain size diameter was measured using EBSD analysis and it was equal to $23 \pm 10 \mu \mathrm{m}$.

A load-controlled fatigue test was performed with a maximum stress of $778 \mathrm{MPa}$ at $\mathrm{R}=0.1$ and $15 \mathrm{~Hz}$. A notch (existing defect) with dimensions of $60 \times 20 \times 4 \mu \mathrm{m}^{3}$ was made on the specimen surface using a focused ion beam to follow its growth. In situ computed tomography (CT) at the BL20XU beamline of SPring- 8 was employed to scan the growing crack on the surface and inside the alloy. The monochromatic X-ray energy of $30 \mathrm{keV}$ with an exposure time of $400 \mathrm{~ms}$, details of the scanning conditions can be found in [9]. The crack was periodically scanned for every $5 \mu \mathrm{m}$ of crack growth projection on the detector, for two different crack front regions. The scanned absorption contrast images were converted into the phase contrast images in order to increase the contrast of gray values between $\alpha$ and $\alpha+\beta$ phases which enhance the binarization of the microstructure [10]. The $\alpha$ grains, their boundaries, and crystallographic orientations were determined and were carefully verified by using the EBSD serial sectioning throughout the specimen. The absorption contrast images were used to determine the crack front coordinates for each slice as compared to the notch; details can be found in [9].

In order to investigate the effect of microstructure on crack growth behavior, a precise analysis of geometrical and crystallographic features was carried out for each cracked grain around the crack front. The cracked grain features were quantified in four classes i.e., shape, size, distribution, and crystallography. The neighboring microstructure interacting with the cracked grain were categorized as the grains in contact, grains within the monotonic, cyclic plastic zones, and grains at the crack front that are given by subscripts $i c, m p, c p$, and $c f$, respectively. The average and maximum features of the neighboring grains are noted by superscript, Max and Ave, respectively. Each class of feature was quantified for those neighboring grains as compared to the cracked $\alpha$ grains. A complete list of the 77 microstructural features can be found in [11]. On the other hand, the average crack growth rate (ACGR) and its variation were quantified for 15 and 38 grains in the first and second region, respectively. Global sensitivity analysis was used to measure the sensitivity of the crack growth behavior along the crack front with respect to the features. The above-mentioned sensitivity was measured via Pearson correlation coefficient (PCC) to rank the microstructural features contributing to the crack growth behavior. Afterwards, principal component analysis (PCA) was used to eliminate the number of microstructural features with similar covariance but lower rankings. Surrogate model was created by considering the highest ranked features to further analyze and to reveal possible microstructural mechanism controlling the short crack path and its variation.

\section{Results}

The reconstructed 3D short crack and the 3D $\alpha$ grains be seen in Figures 1. As it can be seen, crack has a complex morphology with facet and non-facet paths with large deflection angles from the plane perpendicular to the loading direction (z direction) in Figure 1(a). The short crack is surrounded by a total of 318 grains with large variation in size, shape and crystallographic features (Figure 1(b)).

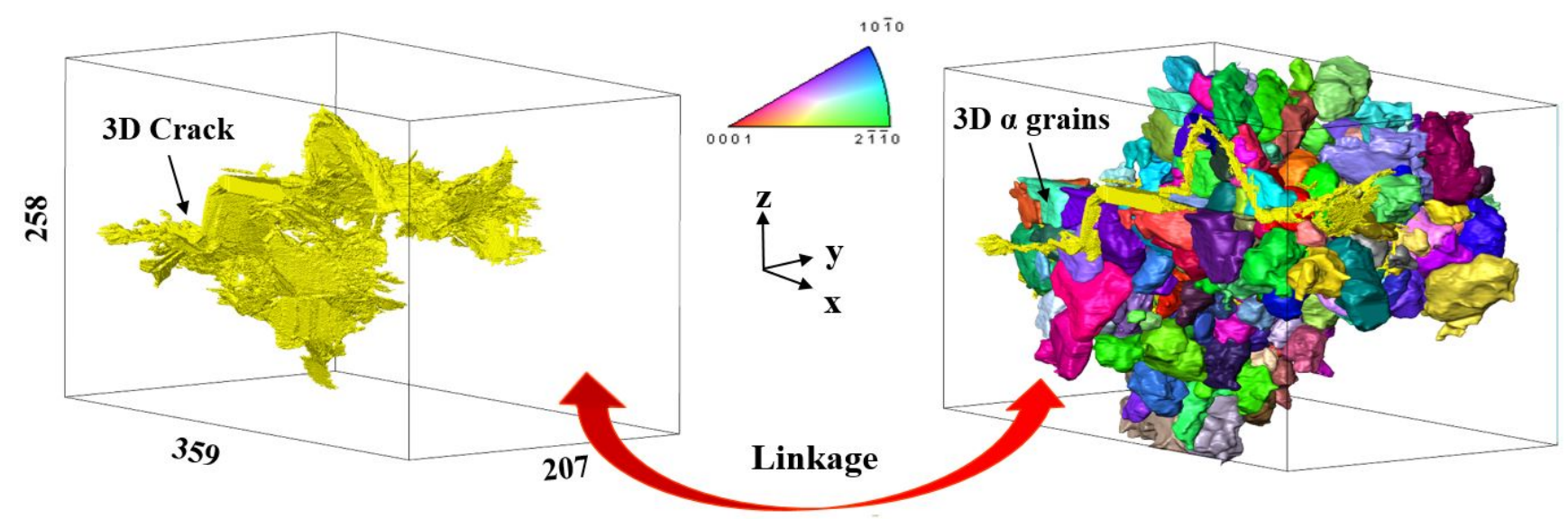

Figure 1. Reconstructed 3D short crack and microstructure in Ti-6Al-4V (grains colors correspond to the IPF map and dimensions are in $\mu \mathrm{m})$. 
Figures 2 show the short crack growth rates inside the microstructure $(\alpha, \alpha+\beta$, and interface $(\alpha / \alpha+\beta))$ along the two crack front regions. The short crack is growing predominantly inside the $\alpha$ grains as compared to the $\alpha+\beta$ phase and interface. The short crack has higher growth rate at some locations, which shows favorable crystallographic paths for crack growth as compared to the other locations along the crack front. As the crack front becomes larger, the crack growth rates increase with a lower variation inside the microstructure (Figure 2(b)), so the crack front shape becomes quasi-homogenous.

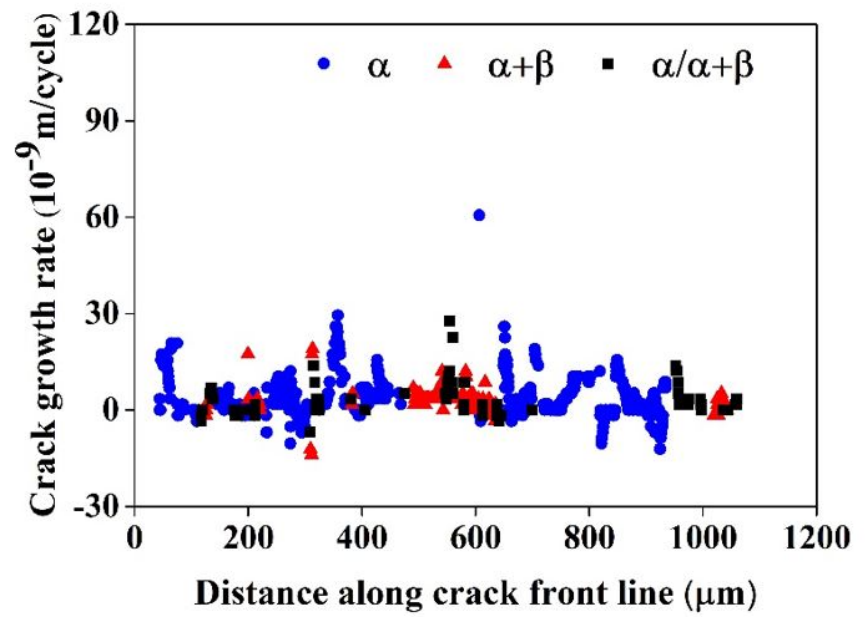

(a)

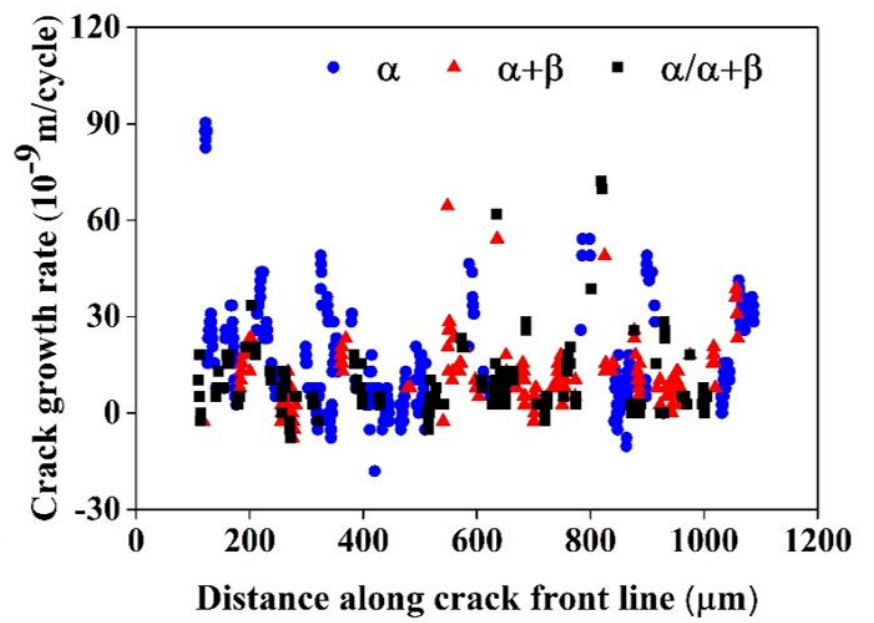

(b)

Figure 2. Short crack growth rate in the microstructure $(\alpha, \alpha+\beta$, and interface $(\alpha / \alpha+\beta))$ along the crack front lines, (a) short crack in the first region, followed by (b) a larger crack front in the second region.

By using the global sensitivity analysis the microstructural features controlling the growing 3D short crack were ranked in both crack front region. For the sake of brevity, only the results for the short crack in facet and non-facet like fracture paths followed by the average short crack growth rate (ACGR) are presented.

The short crack path and its growth rate correlation are shown in Figures 3, the definition of different features can be found in the appendix. The crack path has a stronger correlation with crystallographic features as compared to the other classes of features (encircled features in Figure 3(a)). There is a strong effect of neighboring grains in contact and in the cyclic plastic zone on the short crack path in a facet-like path. The relative difference between the Schmid factor of neighboring grains as compared to the cracked grain leads to crack growth in facet-like paths.

On the other hand, the crack growth rate correlates with the shape, crystallographic, and distribution of the grains (Figure 3(b)). There is competition between these classes of features in order to control the crack growth. The dominant features are the shape of the cracked grains with the distribution of grains in contact ((encircled features in Figure 3(b)).
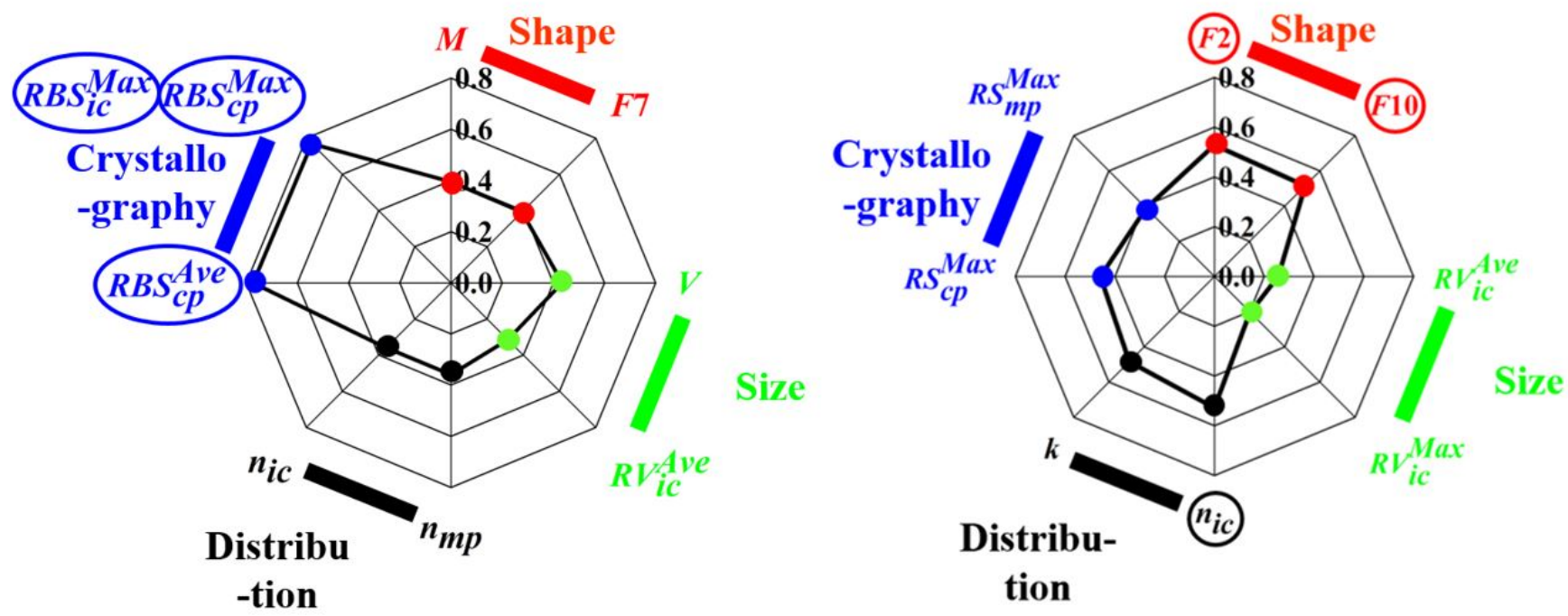
(a)

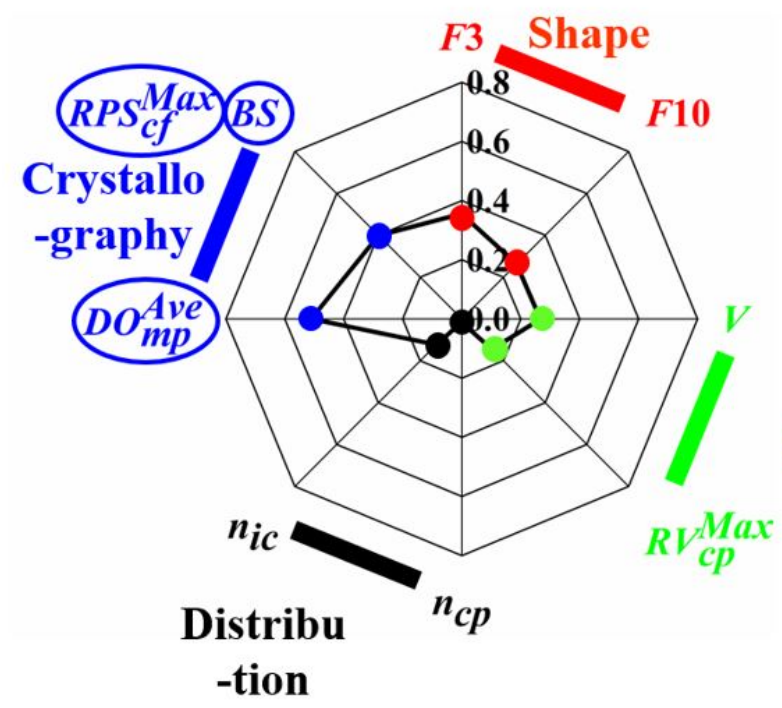

(c) (b)

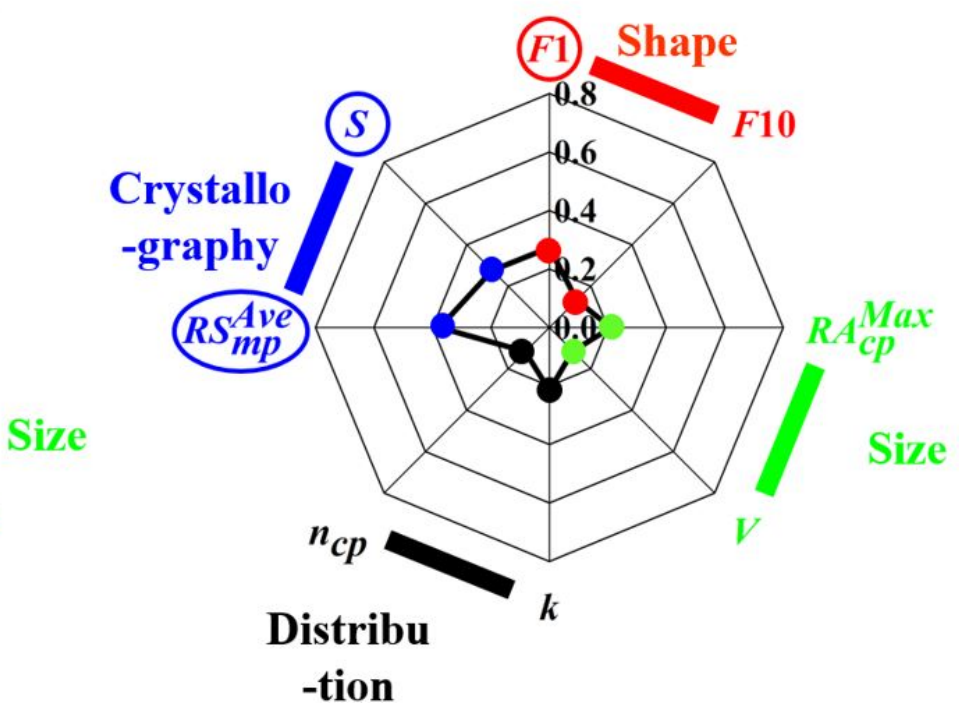

(d)

Figure 3. Pearson correlation graphs for the most contributing features in each category (shape, size, distribution, and crystallography) to the short crack growth behavior. Features controlling (a) crack path, and (b) its growth rate for the short crack. Features controlling the larger crack front with respect to (c) crack path and (d) its growth rate. The encircled features are those with the highest Pearson correlation coefficients.

As the crack front becomes larger, the correlation decreases for both crack path and its growth rate. The crack path is still affected by the crystallographic features of the neighboring grains (encircled features in Figure 3(c)). The neighboring grains at the crack front and the monotonic plastic zones are affecting the crack path. The relative misorientation of those grains with respect to the cracked grain affect the crack path. On the other hand, there is a larger decrease in the correlation with features controlling the crack growth rate. The larger crack front is controlled by the crystallographic features of the cracked grain and neighboring grains and the shape of the cracked grain (encircled features in Figure 3(d)).

The effect of cracked grain and its neighboring grains size on the crack growth behavior is weak in this alloy. Nonetheless, the average diameter of the cracked grain is around $30 \mu \mathrm{m}$, which is higher than the average grain diameter in this alloy $(23 \mu \mathrm{m})$. As the crack front became larger, there is still a tendency to grow inside the grains with larger diameters $(26 \mu \mathrm{m})$ as compared to the average grain diameter. However, large crack paths inside large grains do not necessarily induce high crack growth rate, since the strong interaction with other class of features affects the crack growth behavior.

\section{Discussion}

By creating the surrogate models with the highest ranked features, the relationship between features and crack growth can be studied. Furthermore, the predominant microstructural mechanism controlling the crack growth behavior can be determined. Regarding the crack path, the classifier type of support vector machine were used to determine the relationship between features and crack path.

The short crack grow in a facet like path in grain that in cracked grains that have higher relative basal schmid factor as compared to the neighboring, which are low $R B S_{i c}{ }^{M a x}, R B S_{c p} M a x$, and $R B S_{c p}{ }^{A v e}$ (Figure 4(a)). The possibility of growing in non-facet-like path increase as the basal Schmid factor of neighboring grains in contact and in the cyclic plastic zone increases. It was observed that short crack minimizes its deflection angles with respect to neighboring cracked grain causing variation in crack growth rate.

As the short crack becomes larger, the neighboring grains in the larger monotonic plastic zone and the crack front are affect the crack path. The basal schmid grain of cracked grain is an important factor for a growth in a facet-like path $\left(R P S_{c f} M a x\right.$ and 
$D O_{m p}{ }^{A v e}$ ). Many secondary cracks are formed in the monotonic plastic zones, however, the misorientation with the grains in monotonic plastic zone leads to their arrest.

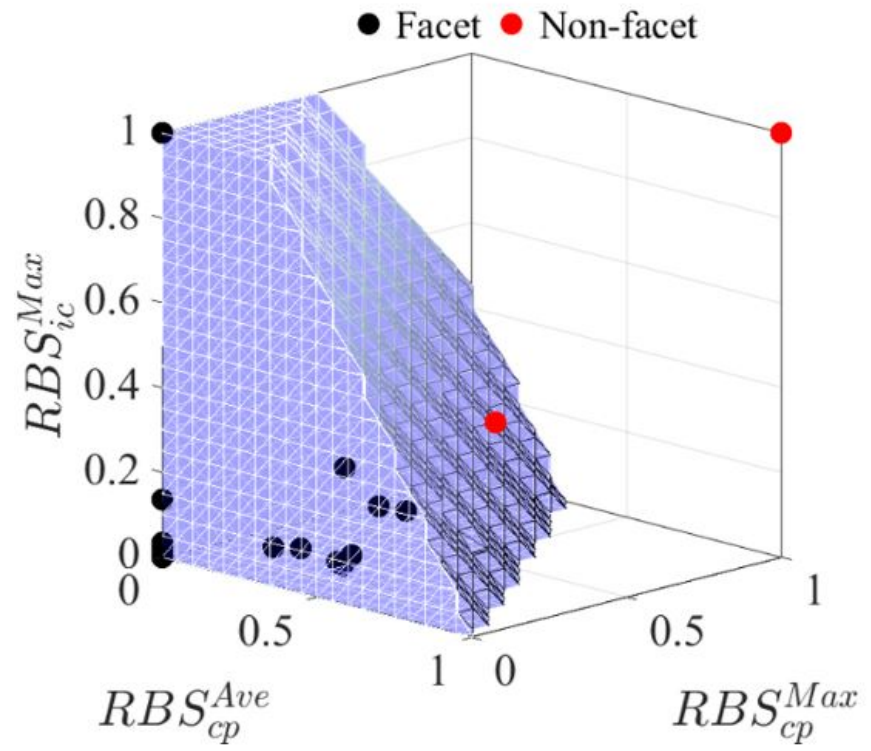

(a)

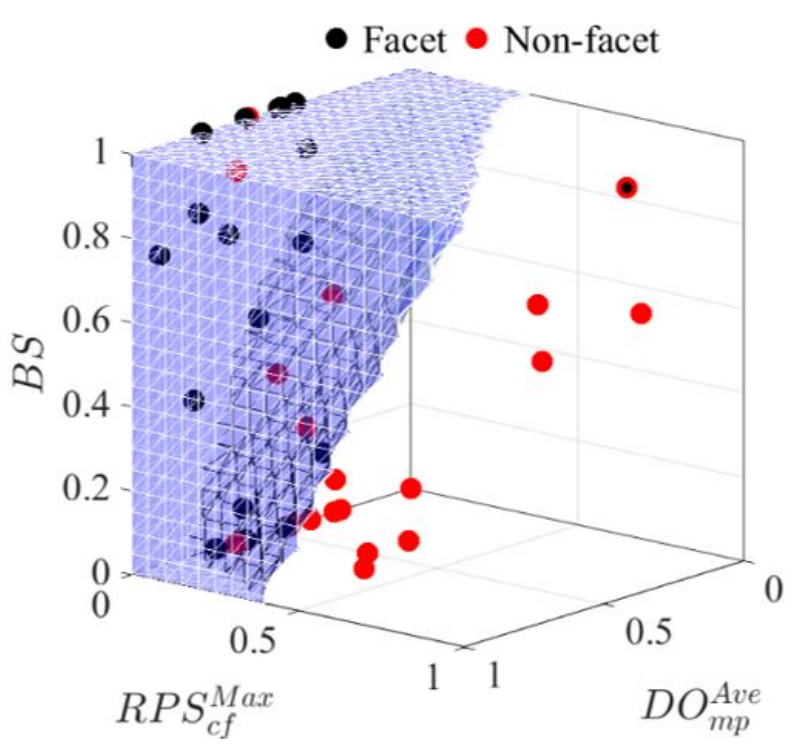

(b)

Figure 4. Three dimensional classification of the most contributing microstructural features correlating with the short crack path as facet and non-facet-like fracture path. (a) The basal schmid of neighboring grains with respect to the cracked grain $\left(R B S_{i c}{ }^{M a x}\right.$, $R B S_{c p}{ }^{M a x}$ and $R B S_{c p}{ }^{A v e}$ ) are the important features for a growth in a facet-like path. (b) As the short crack front becomes larger, a high basal schmid factor of the grain leads to crack growth in facet-like paths but the misorientation with neighboring grains in the monotonic plastic zones $\left(R P S_{c f}{ }^{M a x}\right.$ and $\left.D O_{m p}{ }^{A v e}\right)$.

The 3D response surface curve for ACGR with respect to cracked grain shape $(F 10)$ and grains in contact with it $\left(n_{\mathrm{ic}}\right)$ is shown in Figure 2(a). The data points show that a decrease in F10, which is an increase in grain's length in the loading direction with an increase in number of grains in contact, lead to an optimized (low) ACGR. There is a linear relationship between the features and average crack growth rate with mean squared prediction error of $69 \%$.

As the short crack length increase in the second region, the relationship between the features and average crack growth rate becomes non-linear with mean squared prediction error of $49 \%$. A high average Schmid factor of the grains in the monotonic plastic zone $\left(R S_{m p}{ }^{A v e}\right)$ as compared to the cracked grain elongated along the thickness, results in a formation of crack at secondary location with high shear stress which leads to a decrease in crack driving forces and the ACGR (Figures 2(b)). This stress distribution leads to an increase in crack growth in $\alpha+\beta$ phase and along the interface above the average microstructural fractions. 


\section{- Grain features}

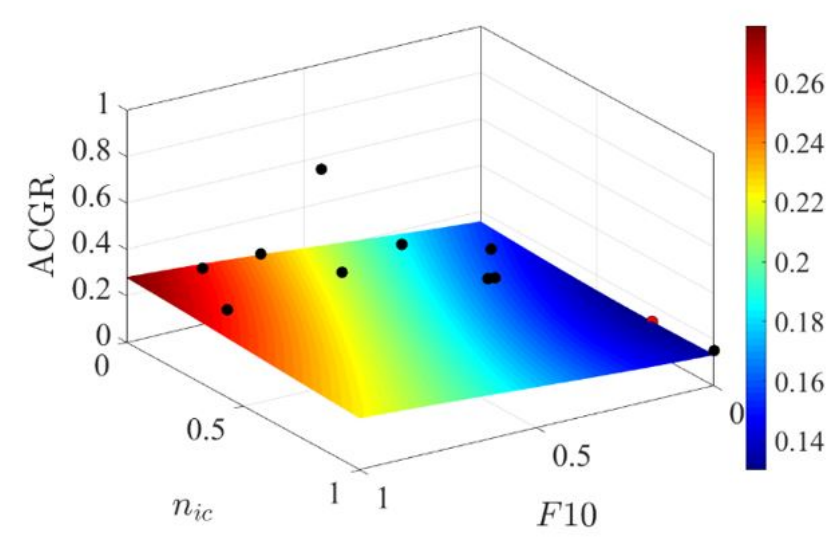

(a)
- Optimum features

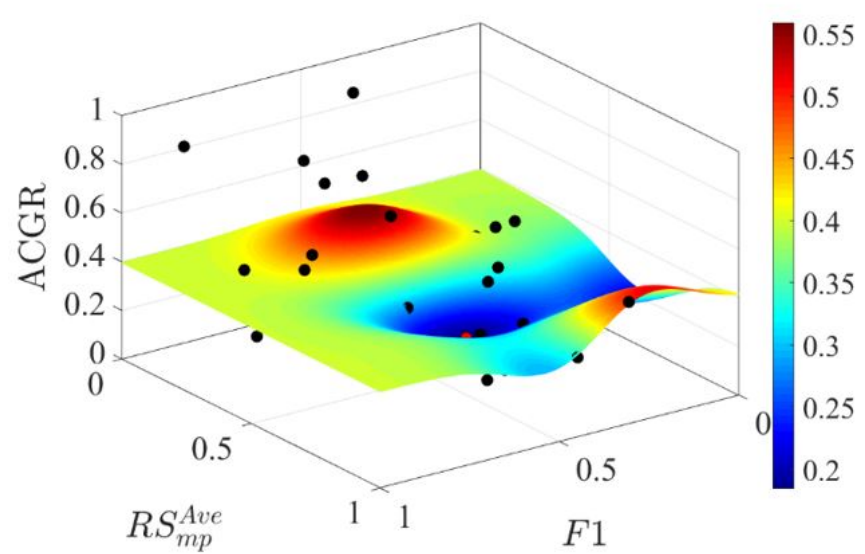

(b)

Figure 5. Three dimensional response surface curve for the most contributing microstructural features correlating with the average crack growth rate (ACGR). (a) Cracked grain elongated in the loading direction (low F10) and grains in contact with it (high $n_{\mathrm{ic}}$ ) lead to low ACGR. (b) As the short crack front becomes larger, a high average Schmid factor of the grains in the monotonic plastic zone $\left(R S_{m p}{ }^{A v e}\right)$ as compared to the elongated cracked grain along the tickness $(F 1)$ lead to low ACGR.

\section{Conclusions}

In this study, the complex 3D microstructure and short crack were characterized and linked in detail by employing the X-ray micro-tomography and serial sectioning using EBSD analysis. Subsequently, the microstructural features contribution to the short crack growth behavior were ranked and coarsened by using global sensitivity and principal component analysis. Afterwards, by creating surrogate models, the relationship between the most contributing microstructural features and short crack growth behavior were further analyzed via support vector machines.

Short crack preferably grows inside the predominant $\alpha$ phase above the average microstructural fraction. The crack plane minimizes the deflection angles with neighboring cracked sections that may lead to growth on a plane with low Schmid factor leading to crack growth rate variation. High number of grains in contact with a long cracked grain in the loading direction impose a constraint on the crack opening that leads to a decrease in ACGR. As the short crack front becomes, high shear stress of the grains in the monotonic plastic zones leads to secondary cracks and crack bifurcation which induces a decrease in crack driving forces at the crack front leading to a decrease in ACGR. This stress distribution leads to an increase in crack growth in $\alpha+\beta$ phase and along the interface above the average microstructural fractions.

\section{Appendix}

Table 1. Description of geometrical and crystallographic features of grains.

\begin{tabular}{|c|c|l|}
\hline Class & Features & Description \\
\hline \multirow{4}{*}{ Shape } & $F 1$ & Elongation along the thickness \\
\cline { 2 - 3 } & $F 2$ & Elongation in loading direction \\
\cline { 2 - 3 } & $F 3$ & Elongation perpendicular to loading direction \\
\cline { 2 - 3 } & $F 7$ & Shape deviation from a cube \\
\cline { 2 - 3 } & $F 10$ & Shape deviation from a cube \\
\hline Size & $V$ & Volume of grain \\
\hline \multirow{2}{*}{ Crystallographic } & $S$ & Apparent maximum Schmid factor \\
\cline { 2 - 3 } & $B S$ & Apparent basal Schmid factor \\
\hline
\end{tabular}


Table 2. Microstructural features of surrounding grains as compared to the cracked grain.

\begin{tabular}{|c|c|c|}
\hline Class & Features & Description \\
\hline \multirow{4}{*}{ Size } & $R V_{i c}^{\text {Ave }}$ & Average volume of grains in contact \\
\hline & $R V_{i c}^{\text {Max }}$ & Maximum volume of grains in contact \\
\hline & $R V_{c p}^{M a x}$ & $\begin{array}{l}\text { Maximum volume of grains in the cyclic plastic } \\
\text { zone }\end{array}$ \\
\hline & $R A_{c p}{ }^{\operatorname{Max}}$ & Maximum area of grains in the cyclic plastic zone \\
\hline \multirow{4}{*}{ Distribution } & $n_{i c}$ & Number of grains in contact \\
\hline & $n_{c p}$ & Number of grains in the cyclic plastic zone \\
\hline & $n_{m p}$ & Number of grains in the monotonic plastic zone \\
\hline & $k$ & Fraction of $\alpha+\beta$ phase \\
\hline \multirow{7}{*}{ Crystallographic } & $R B S_{i c}^{\text {Max }}$ & Maximum Basal Schmid factor of grains in contact \\
\hline & $R B S_{c p} \operatorname{Max}$ & $\begin{array}{l}\text { Maximum Basal Schmid factor of grains in the } \\
\text { cyclic plastic zone }\end{array}$ \\
\hline & $R B S_{c p}$ Ave & $\begin{array}{l}\text { Average Basal Schmid factor of grains in the cyclic } \\
\text { plastic zone }\end{array}$ \\
\hline & $R S_{c p}^{\text {Max }}$ & $\begin{array}{l}\text { Maximum Schmid factor of grains in the cyclic } \\
\text { plastic zone }\end{array}$ \\
\hline & $R S_{m p}^{\operatorname{Max}}$ & $\begin{array}{l}\text { Maximum Schmid factor of grains in the } \\
\text { monotonic plastic zone }\end{array}$ \\
\hline & $R P S_{c p}^{M a x}$ & $\begin{array}{l}\text { Maximum prismatic Schmid factor of grains in the } \\
\text { monotonic plastic zone }\end{array}$ \\
\hline & $D O_{m p}$ Ave & $\begin{array}{l}\text { Average disorientation of grains in the monotonic } \\
\text { plastic zone }\end{array}$ \\
\hline
\end{tabular}

\section{Acknowledgments}

The synchrotron radiation experiments were performed at SPring-8 with the approval of Japan Synchrotron Radiation Research Institute (JASRI) through proposal numbers of 2016A0076, 2016B0076 and, 2017A0076. This work was supported through the grant-in-aid for scientific research from Structural Materials for Innovation (SM4I) of the Cross-ministerial Strategic Innovation Promotion Program (SIP). The authors gratefully acknowledge the support of Light Metal Educational Foundation (LMEF).

\section{References}

[2] and G.B.O. McDowell, David L., in: Sci. Model. Simulations, Springer, Dordrecht, 2008, pp. 207-240.

[3] K. Tokaji, T. Ogawa, K. Ohya, Int. J. Fatigue 16 (1994) 571-578.

[4] A.W. Thompson, W.A. Backofen, Acta Metall. 14A (1971) 597-606.

[5] C.W. Brown, D. Taylor, in: Fatigue Crack Growth Threshold Concepts, 1984, pp. 433-446.

[6] Y. Akiniwa, K. Tanaka, E. Matsui, Mater. Sci. Eng. A 104 (1988) 105-115.

[7] T. Hastie, R. Tibshirani, J. Friedman, Math. Intell. (2008) 764.

[8] A.L. Pilchak, Scr. Mater. 68 (2013) 277-280.

[9] M. Hassanipour, S. Watanabe, K. Hirayama, H. Toda, K. Uesugi, A. Takeuchi, Mater. Sci. Eng. A 738 (2018) $229-237$.

[10] D. Paganin, S.C. Mayo, T.E. Gureyev, P.R. Miller, S.W. Wilkins, J. Microsc. 206 (2002) 33-40. 
[11] K. Uesugi, S. Watanabe, K. Hirayama, A. Takeuchi, H. Toda, H. Li, M. Hassanipour, Mater. Sci. Eng. A 751 (2019) 351362 . 